\title{
Study on Unreamed nailing for long bone fractures.
}

\author{
Dr G. Vara Prasad M.S(Ortho.) ${ }^{1}$, Dr P.Anil Babu M.S(Ortho.) $)^{2}$ \\ ${ }^{I}$ (Department of Orthopaedics, Guntur Medical College ,Guntur ,AP, India) \\ ${ }_{2}^{2}$ (Department of Orthopaedics, Guntur Medical College ,Guntur ,AP, India)
}

\begin{abstract}
Reaming of a long bone has been incriminated in intra operative and post operative complications due to changes in dynamics of intramedullary blood circulation, enormous increase in pressure while reaming can cause damage to the blood vessels and soft tissues which are vital for fracture union. There are situations which present with pre existing comorbid conditions as well as preexisting trauma in the vicinity of the fracture. when nailing has been opted as treatment choice unreamed nailing will have less complications during perioperative period as well as in the long term.
\end{abstract}

Keywords: Long bone fracture, perioperative complications, Unreamed nailing

\section{Introduction}

Preserving blood supply to both soft tissues and bone is crucial in achieving union at the fracture sites. Diaphyseal fractures of the long bones require closed methods to reduce and fix the fractures for preserving fracture haematoma. Fixation techniques like intramedullary nailing is done with both reamed and unreamed nailing. The heat and pressures generated during reaming of the bone can cause damage to the intra medullary blood supply, and also cause dreaded complications like embolism of intramedullary fat. Unreamed nailing has been an option for avoiding possible complications like fat embolism, delayed union, and soft tissue damage. While choosing the unreamed nailing as the technique, it is ensured that the medullary canal is sufficiently filled with the implant for the snug fit at the most narrow part of the medullary canal, isthmus. Usually Tibial fractures are the most common long bone fractures. The aims of fracture treatment for shaft are re-establishing pre-injury anatomy and function along with least complication rates. Several other methods available for treatment of this fracture are compression plating, reamed or unreamed intramedullary nailing and external fixation. Among them, intramedullary nail fixation has been shown to be an effective method for treating both open and closed tibial fractures. The nutrient artery in long bones supplies $62 \%$ of cortical blood flow in the diaphysis and normal blood flow is centrifugal . Intramedullary reaming can destroy the branches nutrient artery and erodes into the endosteal surface of the cortex.

In an uneven medullary canal reamed intramedullary nailing has the advantage of providing optimal biomechanical stability; however, reaming of the medullary canal results in endosteal blood flow damage, bone necrosis, compartment syndrome and infection. In theory, unreamed intramedullary nailing does not have the above-mentioned problems associated with reaming, but the mechanical stability may limit its application. The diaphyses of long bones obtain their arterial blood supply from three sources: the nutrient artery, the metaphyseal arteries and the periosteum ${ }^{[1]}$. In1972, Rhinelander demonstrated that the nutrient artery supplies the inner two-thirds of the cortex and is the chief blood supply to cortical bone, and in 1979 Trias and Fery confirmed this, using Indian ink in mature canine bone. Willans (1987) estimated, by an indicator fractionation technique, that the endosteal blood supply is responsible for up to $62 \%$ of cortical blood flow in the intact bone. The cortical circulation is normally centrifugal.

The vascular system of bone and its reaction to injury are important for fracture healing and have been reviewed by Kelly, Montgomery and Bronk (1990). The measurement of cortical blood flow is difficult because the vascular structure of bone is complex with multiple arterial supplies and venous outflows. Simple ligation of the nutrient artery and the consequent suppression of the normal centrifugal blood flow resulted in a significant reduction in cortical blood flow (Tothill et al 1987; Willans 1987). It did not stimulate an effective compensation by the periosteum. This raises the possibility that the acute haemodynamic response in the periosteum after reaming may not result solely from the absence of the dominant centrifugal blood supply, but may, in part, be an indirect consequence of the additional injury to the endosteal cortex caused by reaming ${ }^{[2]}$.

Both of reamed and undreamed techniques have different advantages on fracture healing: the reamed nailing has a more rigid structure and earlier fracture union, while the unreamed nailing supplies much better blood to the cortex ${ }^{[3]}$.

A number of prospective, randomized controlled trials have compared the effects of reamed and unreamed intramedullary nailing of lower extremity fractures. Meta-analyses of these trials have suggested large reductions in the risk of nonunion, or failure of the fracture to heal, in association with the use of reamed intramedullary nailing (relative risk, $0.44 ; 95 \%$ confidence interval, 0.21 to 0.93 ). Nevertheless, methodological 
limitations, including lack of concealment, blinding, and standardization of care, have left the efficacy of reamed intramedullary nailing uncertain ${ }^{[4]}$.

This study has been designed to involve diaphyseal fractures of different long bones with different intramedullary devices.

\section{Materilas And Methods}

This study has included diaphyseal fractures of long bones which are closed and Type I open fractures of Gustillo Andersons. All the fractures were initially stabilized to assess the fitness to undergo surgery. Diaphyseal fractures of Femur, Tibia, Humerus, Radius and Ulna are involved in the study. Some of the closed fractures have skin of doubtful viability though there was no breach of skin. It has been taken as inclusion criterion to involve open fractures as mentioned above, which did not show periosteal breach. All the fractures involved in the study were with transverse to short oblique in the fracture pattern. Another inclusion criterion was fractures without any comminution. The patients included in the study did not show any co morbid conditions, which was evaluated thoroughly before posting for surgery. It has been ensured that the fractured limbs did not have any skin breaches in the vicinity of the entry points used for insertion of the Bone awls and intramedullary nails. All the cases are observed for skin changes at the site of the entry point and fracture for any damage due to trauma inflicted on the skin and underlying soft tissues. At the same time the fracture site is examined for any colour changes, increase in swelling, redness, pain related to operative trauma as perceived by the patient.

\section{Surgical Technique And Post Operative Followup}

All the patients included in this study have undergone different types of internal fixation, essentially after closed reduction of the fracture. This aspect ensures that the fracture site did not receive any trauma afresh apart from initial trauma to observe the effect of unreamed nailing on fracture. All the entry points are made on intact skin only. The diameter of the intramedullary devices are measured pre and peri operatively to fill the unreamed medullary canal for the snug fit, like what could be achieved by reamed nailing. The narrowest part of the long bone has been measured with intramedullary device kept on the skin under image intensifier. The fractures were reduced with traction and counter traction, and also on fracture table. Different intramedullary devices used are square nails, Enders nails, TENS, Solid nails.

The patients are followed up with three dressing changes in the immediate post operative period to observe the skin changes and swelling, redness. The suture removal at the entry points and Type I open fracture site were done at tenth post operative day. First three post operative radiographs are taken with four weeks gap, after second day radiograph. Gentle supported ROM was advised from third post operative week onwards.

The study and results has involved 108 patients of long bone fractures who have completed a minimum post operative followup of six months. All the patients had good wound healing with dry surgical wounds at tenth post operative day. The ROMs retained by the patient are assessed from sixth post operative week for upper limbs and eighth to twelfth for lower limb fractures.

\section{Figures And Tables}

Table 1

\begin{tabular}{|l|l|l|l|l|l|l|}
\hline & Nature & Pattern & Implant & Reduction & Functional activity & No.: \\
\hline Femur & Closed & Transverse & Enders & Closed & 12 weeks & 21 \\
\hline Tibia & Type I GA & Short oblique & Solid ILN & Closed & 12 weeks & 42 \\
\hline Humerus & Closed & Transverse & Enders & Closed & 6 weeks & 8 \\
\hline Radius & Closed & Transverse & Square Nail & Closed & 6 weeks & 14 \\
\hline Ulna & Type I GA & Short oblique & Square Nail & Closed & 6 weeks & 23 \\
\hline
\end{tabular}

Table 2

\begin{tabular}{|l|c|c|c|}
\hline & Dressing Changes & Suture removal & Assisted Movements \\
\hline Femur & 3 & $10-14$ days & Seventh Post Op day \\
\hline Tibia & 3 & $10-14$ days & Seventh Post Op day \\
\hline Humerus & 3 & $8-10$ days & Fifth Post op day \\
\hline Radius & 3 & $8-10$ days & Fifth Post op day \\
\hline Ulna & 3 & $8-10$ days & Fifth Post op day \\
\hline
\end{tabular}



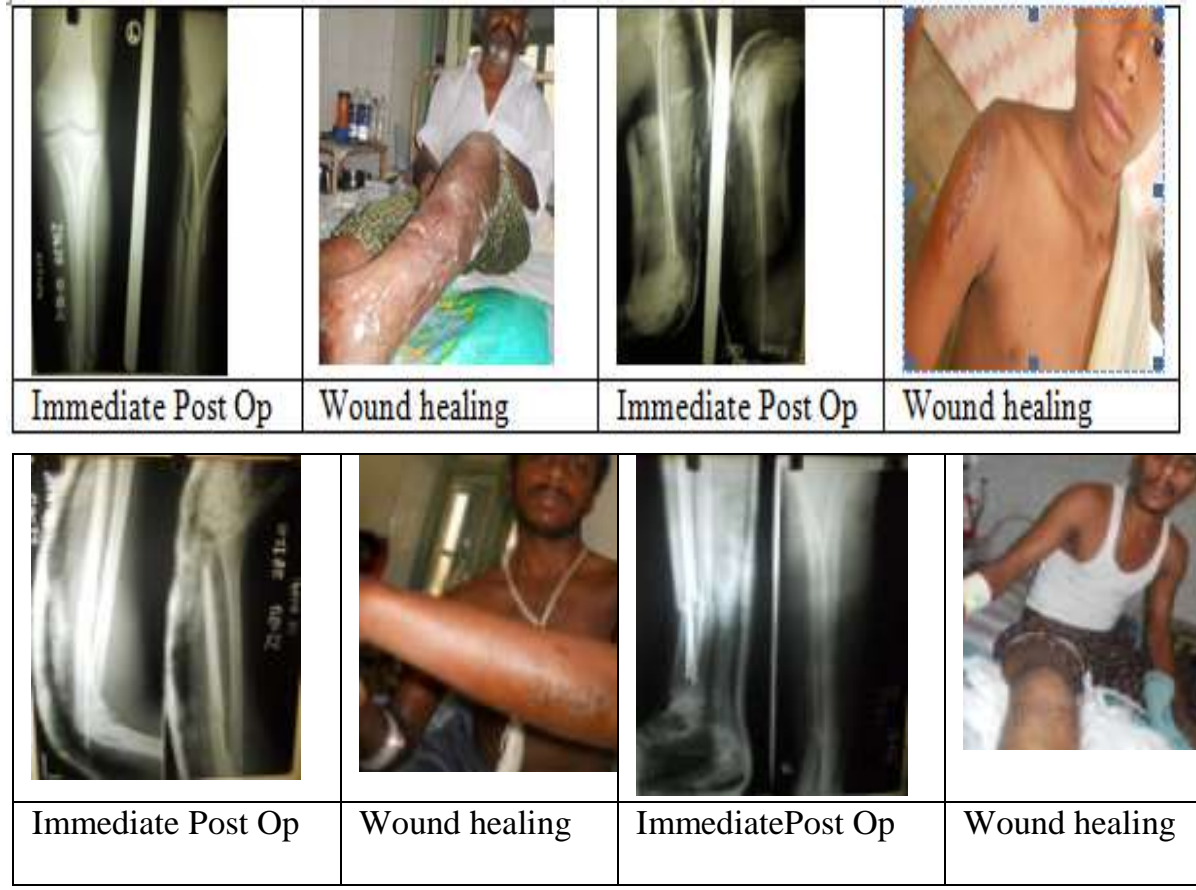

\section{Conclusion}

Unreamed interlocking, Enders nail fixation, TENS, Square nail will have less tissue damage. The beneficial effects are required when soft tissue viability is doubtful. The swelling, redness in the pre operative period considerably come down in unreamed nailing. Open fractures without periosteal breach can be safely managed with undreamed nailing. Undreamed nailing consumes shorter operative time. All possible complications can be minimized by adopting undreamed technique. Minimal tissue damage helps in early recovery and to regain ROM.

\section{References}

Journal Papers:

[1]. Deting Xue \& Qiang Zheng \& Hang Li \& Shengjun Qian \& Bo Zhang \& Zhijun Pan Reamed and unreamed intramedullary nailing for the treatment of open and closed tibial fractures: a subgroup analysis of randomised trials

[2]. The acute vascular response to intramedullary reaming - microsphere estimation of blood flow in the intact ovine tibia. L. H. REICHERT. I. D. MCCARTHY, S. P. F. HUGHES From the Royal Postgraduate Medical School, Hammersmith Hospital, London, England.

[3]. Meta-analysis of reamed versus undreamed intramedullary nailing for open tibial fractures Yinchu Shao, Hongxing Zou*, Shaobo Chen and Jichun Shan. Shao et al. Journal of Orthopaedic Surgery and Research 2014, 9:74.

[4]. Randomized Trial of Reamed and Unreamed Intramedullary Nailing of Tibial Shaft Fractures By the Study to Prospectively Evaluate Reamed Intramedullary Nails in Patients with Tibial Fractures (SPRINT) Investigators* Investigation performed at McMaster University, Hamilton, Ontario, Canada. J Bone Joint Surg Am. 2008;90:2567-78.

[5]. A Prospective Comparative Study of Reamed vs. Unreamed Nailing in Fractures Shaft of Tibia Malays Orthop J. 2012 Nov; 6(3): 21-26. doi: 10.5704/MOJ.1207.0016 PMCID: PMC4093593 ,Dinesh Choudary, B Kanthimathi

[6]. Reamed versus unreamed femoral nails A RANDOMISED, PROSPECTIVE TRIAL M. G. Clatworthy, D. I. Clark, D. H. Gray, A. E. Hardy From Auckland and Middlemore Hospitals, Auckland, New Zealand, 1998 British Editorial Society of Bone and Joint Surgery ,0301-620X/98/37493, VOL. 80-B, NO. 3, MAY 1998. 